Onkologie 1983;6:213

\title{
Impressum, Vol. 6, No. 5, 1983
}

\section{S. KARGER}

Verlag für Medizin und Naturwissenschaften GmbH, Postfach 2, D-8034 Germering

\section{Wissenschaftlicher Beirat}

H. W. Bauer, München; H. Denck, Wien; V. Diehl, Hannover; F. Douwes, Göttingen; P. Drings, Heidelberg; S. Eckhardt, Budapest; H. Ehrhart, München; W. M. Gallmeier, Nürnberg, H. Gerhartz, Berlin; E. H. Graul, Marburg/Lahn; R. Gross, Köln; H. Heimpel, Ulm; K. P. Hellriegel, Köln; Ch. Herfarth, Ulm; J. H. Holzner, Wien; R. Hünig, Basel; W. Hunstein, Heidelberg; H. O. Klein, Köln; H. Löffler, Kiel; U. Mohr, Hannover; F.-L. Müller, Bonn; K. Munk, Heidelberg; G. P. Murphy, Buffalo (N.Y.); G. A. Nagel, Göttingen; A. Pfleiderer, Freiburg; Mildred Scheel, Bonn; E. Scherer, Essen; S. Seeber, Essen; H. J. Senn, St. Gallen; A. Stacher, Wien; St. Tanneberger, Berlin/DDR; * W. Vahlensieck, Bonn; H. D. Waller, Tubingen; M. Wannen-macher, Freiburg; W. Wilmanns, München; K. Wilms, Würzburg; K. zum Winkel, Heidelberg; H. Wrba, Wien.

Verantwortliche Schriftleitung z. Zt. S. Eckhardt, Budapest J. H. Holzner, Wien G. A. Nagel, Göttingen

Die Zeitschrift erscheint zweimonatlich; pro Jahr erscheint 1 Band zu je 6 Heften.

Bezugspreis für Jahrgang 6, 1983 DM 124,-/öS 868,-/SFr. 124,-. 1 Einzelheft kostet DM 26,-/öS 182,-/SFr. 26,-, einschließlich MwSt., zuzüglich Postgebühren.

Der Abonnementspreis ist im voraus zahlbar. Die Lieferung der Zeitschrift läuft weiter, wenn sie nicht spätestens 4 Wochen vor Abschluß eines Bandes abbestellt wird.

Abonnementsbestellungen können bei jeder Buchhandlung, bei der Post oder direkt beim Verlag aufgegeben werden: Bundesrepublik Deutschland/õsterreich: S. Karger GmbH, Postfach 2, D8034 Germering/München, Tel: (089) 844021, Telex: 524865 D, Postscheckkonto: München 400 80-807 Schweiz: S. Karger AG, Allschwilerstr. 10, Postfach, CH-4009 Basel, Tel.: (061) 3908 80, Telex $62652 \mathrm{CH}$

\section{Anzeigen}

S. Karger Verlag für Medizin und Naturwissenschaften GmbH, Postfach 2, 8034 Germering, Telefon (0 89) 844021.

Für den Inhalt außerhalb des redaktionellen Teiles (insbesondere Anzeigen, Industrieinformationen, Pressezitate und Kongreßinfor-mationen) übernehmen Herausgeber und Verlag keine Gewähr.

Alle Rechte, insbesondere das Recht der Vervielfältigung und Mikrokopie sowie der Übersetzung, vorbehalten. Nachdruck, auch auszugsweise, nur mit Genehmigung des Verlages.

(C) Copyright 1983 by S. Karger Verlag für Medizin und Naturwissenschaften GmbH, Postfach 2, D-8034 Germering

Satz und Druck: Walter Biering GmbH, Grafischer Betrieb, 8000 München 45

Offizielles Organ

der Deutschen Gesellschaft für Hämatologie und Onkologie 
und der

österreichischen Gesellschaft für Hämatologie und Onkologie Offizielles Organ

der österreichischen Krebsgesellschaft - Krebsliga 